\title{
Origin of Meyer-Neldel type compensation behavior in organic semiconductors at large carrier concentrations: Disorder versus thermodynamic description
}

\author{
I. I. Fishchuk, ${ }^{1,2}$ A. Kadashchuk, ${ }^{2,3, *}$ A. Mityashin, ${ }^{3}$ M. M. Gavrilyuk, ${ }^{1}$ A. Köhler, ${ }^{4}$ H. Bässler, ${ }^{4}$ J. Genoe, ${ }^{3}$ \\ H. Sitter, ${ }^{5}$ and N. S. Sariciftci ${ }^{6}$ \\ ${ }^{1}$ Institute for Nuclear Research, National Academy of Sciences of Ukraine, Prospect Nauky 47, 03028 Kyiv, Ukraine \\ ${ }^{2}$ Institute of Physics, National Academy of Sciences of Ukraine, Prospect Nauky 46, 03028 Kyiv, Ukraine \\ ${ }^{3}$ IMEC, Kapeldreef 75, B-3001 Leuven, Belgium \\ ${ }^{4}$ Experimental Physics II and Bayreuth Institute of Macromolecular Research (BIMF), Universitätsstrasse 30, 95448 Bayreuth, Germany \\ ${ }^{5}$ Institute of Semiconductor \& Solid State Physics, Johannes Kepler University of Linz, A-4040 Linz, Austria \\ ${ }^{6}$ Linz Institute for Organic Solar Cells (LIOS), Johannes Kepler University of Linz, A-4040 Linz, Austria
}

(Received 25 June 2014; revised manuscript received 21 August 2014; published 1 December 2014; corrected 24 April 2015)

\begin{abstract}
We have extended an effective medium approximation theory [Fishchuk, Kadashchuk, Genoe, Ullah, Sitter, Singh, Sariciftci, and Bässler, Phys. Rev. B 81, 045202 (2010)] to investigate how polaron formation affects the Meyer-Neldel (MN) compensation behavior observed for temperature-dependent charge-carrier transport in disordered organic semiconductors at large carrier concentrations, as realized in organic field-effect transistors (OFETs). We show that the compensation behavior in organic semiconductor thin films can be consistently described for both nonpolaronic and polaronic hopping transport in the framework of the disorder formalism using either Miller-Abrahams or polaron Marcus rates, respectively, provided that the polaron binding energy is small compared to the width of the density of states (DOS) distribution in the system. We argue that alternative models based on thermodynamic reasoning, like the multiexcitation entropy (MEE) model, which assumes charge transport dominated by polarons with multiphonon processes and ignores the energy disorder, are inherently not applicable to describe adequately the charge-carrier transport in disordered organic semiconductors. We have suggested and realized a test experiment based on measurements of the compensation behavior for the temperature-dependent conductivity and mobility in OFET devices to check the applicability of these models. We point out that the MN behavior observed in thin-film OFETs has nothing to do with the genuine MN rule predicted by the MEE approach, but rather it is an apparent effect arising as a consequence of the functional dependence of the partial filling of the DOS in a disordered system with hopping transport. This fact is fully supported by experimental results. The apparent MN energy was found to depend also on the shape of the DOS distribution and polaron binding energy.
\end{abstract}

DOI: 10.1103/PhysRevB.90.245201

PACS number(s): 72.20.Ee, 72.80.Le, 72.80.Ng, 71.38.-k

\section{INTRODUCTION}

Organic semiconductor films offer potential for emerging flexible large-area electronics, because they allow low-cost and low-temperature device fabrication compatible with flexible plastic substrates [1,2]. In spite of a large amount of work having been done on the description of charge-carrier transport in organic materials over the last decades, processes that determine such charge transport in realistic organic electronic devices are still not completely understood. Their comprehension, however, is definitely a key to rational material design and, thereby, to further increase of device performance. The commonly accepted mechanism of charge-carrier transport in disordered organic semiconductors is thermally activated hopping through a manifold of localized states distributed in space and energy (see Refs. [3-8] and references therein). The density of state (DOS) distribution in organic disordered solids is commonly accepted to be of a Gaussian shape. The Gaussian disorder (GD) in combination with the Miller-Abrahams (MA) hopping rate model [9] has been the most widely used formalism in the past decades to describe the charge transport in small-molecule and polymer-semiconducting films [3-8]. One reason for the wide acceptance of the GD model is the suc-

*kadash@iop.kiev.ua cessful numerical reproduction of experimental results in many different organic materials and electronic devices. There have been several important improvements of the initial GD model of Bässler [3]; one of the recent prominent advancements is accounting for the partial DOS filling in the presence of space charge to relate charge-carrier concentration and mobility. The latter has spurred the development of a so-called extended Gaussian disorder (EGD) model [10-14], which is currently commonly applied to describe the charge-carrier mobility in organic field-effect transistors (OFETs) and organic spacecharge-limited diodes. The practical applicability of the EGD approach for organic semiconductors is further proved by its wide adoption in commercial device simulation software packages.

Recently, Fishchuk et al. [15] applied an effective medium approximation (EMA) theory in the framework of the EGD model to consider the so-called Meyer-Neldel (MN) compensation effect often observed experimentally for the chargecarrier mobility in thin-film OFETs [16]. This EMA approach was also used before to describe the carrier concentration dependence [14] and electric field dependence [17,18] of the OFET mobility in disordered organic semiconductors. The analytic model demonstrated a perfect agreement with the relevant computer simulation results $[11,12]$ obtained for the same material parameters and available experimental data. It should be mentioned that originally the $\mathrm{MN}$ compensation rule was 
suggested in the 1930s as an empirical relation derived from chemical kinetics [19]. Generally, it states that in a thermally activated process $R(T)$, the increasing activation energy $E_{a}$ is partially compensated by an increasing prefactor so that $R(T)=R_{\text {const }} \exp \left(E_{a} / k_{B} T_{\mathrm{MN}}\right) \times \exp \left(-E_{a} / k_{B} T\right)$, where $T_{\mathrm{MN}}$ is the so-called characteristic $\mathrm{MN}$ temperature. The major results of the EMA-based EGD model [15], relevant to the $\mathrm{MN}$ compensation behavior under consideration in the present paper, are the following:

(i) It was predicted that the $\mathrm{MN}$ compensation effect should be observable in disordered organic semiconductors only if the carrier concentration is sufficiently large [15], viz. when there is a partial filling of the DOS like in OFET devices, and it should not be detectable in a small-carrierconcentration transport regime, as for instance in time-offlight (ToF) measurements. The compensation effect was experimentally observed for the temperature dependences of the OFET mobility $\mu(T)$ upon varying the charge-carrier concentration, but it was not found in experiment by varying the width of the DOS [20]. These two facts led to the important conclusion that the MN type compensation effect observed in organic semiconductors is an apparent rather than a genuine MN rule [15]. Thus, with respect to the temperature-dependent OFET mobility, it would be more correct to call it a "compensation behavior," as it will be used hereafter in this paper.

(ii) The EGD model predicts that the isokinetic temperature (so-called MN energy $E_{\mathrm{MN}}=k_{B} T_{\mathrm{MN}}$ ), obtained for OFET mobility, is related to the width of the Gaussian DOS, $\sigma$, providing thus a method for evaluating the amount of energetic disorder in the material $[15,20]$, which could have a practical importance. All the above mentioned predictions of the EGD model have been verified by experimental studies of $\mathrm{C}_{60}$-based OFET devices [20-22].

Generally, in the context of semiconductor physics, the MN compensation phenomenon has often been observed in thinfilm transistors (e.g., in amorphous $\mathrm{Si}$, chalcogenide glasses, oxide semiconductors); however, the microscopic origin of the effect and its physical meaning in disordered semiconductors are still topics of discussions. Various theoretical explanations suggested before can be divided into two main categories.

The first one, which invokes the concept of energy disorder, has been more traditionally used to describe the MN phenomenon in disordered inorganic transistors. Among these models, the so-called statistical shift of the Fermi level (SSF) model [23-26] is the most elaborated approach and most commonly known as the origin of the MN effect for electron transport in a-Si:H. The basic idea of the SSF model is quite obvious: Thermally activated charge-carrier transport in disordered semiconductors arises due to charge-carrier hopping from the Fermi level to the mobility edge [27], and since the Fermi level shifts with temperature ("the statistical shift") as a consequence of the asymmetry of the DOS around it, the activation barriers that need to be overcome by charge-carrier hopping would also change with temperature, which immediately results in a kind of compensation effect. The SSF models typically consider exponential DOS and MA jump rates, and they also attribute the observed charge-carrier mobility to the width of the DOS $\left(k_{B} T_{\mathrm{MN}}=E_{0}\right.$, where $E_{0}$ is the width of exponential distribution) in semiconductor materials. The analytical EMA-based EGD model $[14,15]$ also belongs to the category of disorder models, with several major modifications especially relevant to organic materials. It assumes a Gaussian-shaped DOS and considers the charge-carrier hopping from a broad equilibrium distribution of occupied localized states, where the carriers migrating within a Gaussian DOS settle; i.e., it accounts for the whole occupational DOS (ODOS) distribution and not just a discrete Fermi level as does the SSF model. The EGD model [14,15] has also incorporated the concept of the effective transport energy level, which is reminiscent of the mobility edge in disordered inorganic semiconductors.

The second category of alternative models is based on thermodynamic reasonings. Yelon and Movaghar [28] and Yelon et al. [29] pointed out that enthalpy and entropy in thermally activated kinetic processes are proportional. They suggested the so-called multiexcitation entropy (MEE) model, which considers the transition between two sites with a difference in energies $\varepsilon_{2}-\varepsilon_{1}=E_{a}$ (a two-site system). The $\mathrm{MN}$ compensation effect in their model arises as a result of multiphonon activated transitions, while no such a behavior is expected for single phonon transitions. An important requirement of the MEE model, for the MN compensation effect to occur, is large values of the activation energies (they indicated $E_{a} \sim 1 \mathrm{eV}$ [28]). According to the MEE model, the multiphonon excitations result in an exponential increase of the number of different activation paths and consequently in an exponential increase of the prefactor with increasing $E_{a}$. This brought the authors to the interpretation of the characteristic temperature as the excitation energy divided by a coupling constant [29,31]. The authors eventually made an ambitious claim that their MEE model offers a universal explanation for the $\mathrm{MN}$ compensation effect for thermally activated phenomena, irrespective whether they take place in disordered or crystalline solids, in biology, in chemical catalytic reactions, and even in geology (see Ref. [31] and references therein). They claim that the $\mathrm{MN}$ effect should always arise whenever the activation energy of a thermally activated process is sufficiently large. In addition, Yelon and Movaghar [28] and Emin [30] claim that in order to yield the $\mathrm{MN}$ effect, the electron transport in a material should be dominated by polarons with multiphonon processes.

Recently, the debate on the applicability of the abovementioned models to describe the MN behavior in disordered semiconductors has been revived by Yelon [31]. It was argued that (i) in general, the disorder formalism, including SSF models, was not applicable to explain MN phenomena at all, and leads to contradictions because of the use of the MA hopping rates, and (ii) the EGD model was not appropriate to experimental situations in OFETs and, therefore, the MN temperature could not be attributed to a measure of disorder. Instead, it was proposed that the observed compensation phenomena in organic semiconductors can readily be explained by the MEE model [28,29], which is the best one to provide a correct and full description of the temperature-dependent charge-carrier mobility in OFET devices.

The present paper reinvestigates the problem by thorough consideration of polaron effects on the $\mathrm{MN}$ behavior of the charge transport in disordered organic semiconductors. We find, that although the MEE model might describe the "genuine 
MN compensation rule," it is inapplicable to the "apparent" MN-like compensation behavior observed in OFETs. The compensation behavior in OFETs is dominated by disorder effects and can be described within the EGD formalism for both nonpolaronic and polaronic hopping transport. We show that the recovered MN compensation behavior is virtually independent of whether MA [9] or Marcus rates [32,33] are used, unless the polaron activation energy is much larger than the energy disorder parameter. In the current paper, we report on test experiments in which we are able to distinguish between the applicability of the EGD and MEE models to OFETs. We found that the isokinetic $T_{\mathrm{MN}}$ temperature is distinctly different for mobility and conductivity, featuring normal- and inverted-MN effects, respectively, which naturally follow from hopping transport within a Gaussian DOS distribution. At the same time, the MEE model predicts a single $T_{\mathrm{MN}}$ for the same material. The experimental results were found to be in good agreement with the present theory. We focus here only on organic semiconductors; other situations where the MN behavior is observed are beyond the scope and ambition of this paper.

\section{THEORETICAL FORMULATIONS}

We consider a disordered organic solid containing localized sites with an average intersite distance $a=N^{-1 / 3}$, where $N$ is the concentration of the localized states. Charge-carrier transport in such a material occurs by thermally assisted hopping. The MA hopping rate $W_{i j}$ for a charge carrier between starting $\varepsilon_{i}$ and target $\varepsilon_{j}$ states has been suggested for a rigid matrix devoid of any polaron effects and is given as [9]

$$
\begin{aligned}
& W_{i j}=W_{1} \exp \left[-\frac{\left|\varepsilon_{j}-\varepsilon_{i}\right|+\left(\varepsilon_{j}-\varepsilon_{i}\right)}{2 k_{B} T}\right], \\
& W_{1}=v_{0} \exp \left(-2 \frac{a}{b}\right),
\end{aligned}
$$

where $v_{0}$ is the attempt-to-escape frequency, and $a$ and $b$ are the intersite distance and carrier localization radius, respectively. The MA model is conventionally based on a single-phonon approximation. On the other hand, the Marcus jump rates are most often applied to account also for polaron formation in organic materials in terms of the nonadiabatic small-polaron concept given by the Marcus theory [32,33]

$$
\begin{aligned}
& W_{i j}=W_{2} \exp \left[-\frac{\varepsilon_{j}-\varepsilon_{i}}{2 k_{B} T}-\frac{\left(\varepsilon_{j}-\varepsilon_{i}\right)^{2}}{16 E_{a}^{\mathrm{pol}} k_{B} T}\right], \\
& W_{2}=W_{0} \exp \left(-\frac{E_{a}^{\mathrm{pol}}}{k_{B} T}\right),
\end{aligned}
$$

where $E_{a}^{\mathrm{pol}}$ is the polaron activation energy, which is equal to half of the polaron binding energy $\left(E_{a}^{\mathrm{pol}}=E_{p} / 2\right), W_{0}=$ $\left(J_{0}^{2} / \hbar\right) \sqrt{\pi / 4 E_{a}^{\mathrm{pol}} k_{B} T} \exp (-2 a / b)$, and $J_{0}$ is prefactor in the transfer integral.

We focus here on comparative consideration of the temperature dependences of the hopping charge transport upon varying carrier concentrations for nonpolaronic and polaronic charge transport described by MA and Marcus jump rates, respectively, in a three-dimensional (3D) disordered organic system. For a given carrier concentration $n$, the Fermi energy level $\varepsilon_{F}$ is determined from the transcendental equation

$$
n=\int_{-\infty}^{\infty} d \varepsilon g(\varepsilon) f\left(\varepsilon, \varepsilon_{F}\right)
$$

where $f\left(\varepsilon, \varepsilon_{F}\right)$ is given by the Fermi-Dirac statistics

$$
f\left(\varepsilon, \varepsilon_{F}\right)=\frac{1}{1+\exp \left(\frac{\varepsilon-\varepsilon_{F}}{k_{B} T}\right)} .
$$

Unless otherwise indicated, we use in this paper a Gaussian DOS $g(\varepsilon)$ distribution with variance $\sigma$, which is also called the energy disorder parameter

$$
g(\varepsilon)=\frac{N}{\sigma \sqrt{2 \pi}} \exp \left[-\frac{1}{2}\left(\frac{\varepsilon}{\sigma}\right)^{2}\right], \quad-\infty<\varepsilon<\infty .
$$

It should be mentioned that a previous EMA treatment [14] considered either nonpolaronic (MA) mobility [15] or the "specific" case of polaronic transport in a Gaussian DOS distribution when polaron effects are much larger than the disorder effects $\left(E_{a}^{\mathrm{pol}} \gg \sigma\right)$. In the latter case, the Marcus jump rate could be readily reduced to a simpler so-called "symmetric" jump rate equation (where the quadratic term in Eq. (2) is ignored [14]). Unfortunately, the previous EMA theory [14] was inherently unable to treat the case of relatively small polaron formation energies, which is more relevant to realistic situations in organic semiconductors normally featuring small or moderate polaronic effects. Besides, we should note that no $\mathrm{MN}$ compensation effect regarding the temperature dependences of the charge-carrier mobility upon varying the carrier concentration could be recovered by EMA theory when $E_{a}^{\mathrm{pol}} \gg \sigma$ because of a vanishing carrier-concentration dependence for the polaron transport [14] at large polaron formation energy (see also discussion hereinafter). Thus, the MN behavior was never considered before by the EMA treatment for polaronic transport in organic semiconductors.

The present paper is based on a different and more advanced EMA formalism formulated for the full Marcus jump rate equation [Eq. (2)] and arbitrary $E_{a}^{\mathrm{pol}} / \sigma$ ratios, i.e., including also relatively weak polaronic effects; therefore, it goes beyond the aforementioned limitations of Ref. [14]. A key aspect of the present theoretical treatment is that the effective charge mobility is determined here from the effective conductivity as

$$
\mu_{e}=\frac{\sigma_{e}}{e n}
$$

where concentration $n$ is given by Eq. (3). The conductivity can be calculated by the EMA method suggested earlier by Kirkpatrick [34]. The principal advantage of this approach over a direct calculation of the charge-carrier mobility [14] is that it can also be applicable for the description of polaronic transport at arbitrary polaron activation energy $E_{a}^{\mathrm{pol}}$ with respect to the energy disorder parameter $\sigma$, since it avoids shortcomings involved in the effective transport energy concept. It should be mentioned that calculation of the transport energy is very problematic for the Marcus jump rates given by Eq. (2) [35]. 
According to Kirkpatrick [34], the effective conductivity $\sigma_{e}$ is determined as

$$
\left\langle\frac{\sigma_{12}-\sigma_{e}}{\sigma_{12}+2 \sigma_{e}}\right\rangle=0
$$

where $\sigma_{12}=G_{12} / a$ is the conductivity in two-site cluster approximation, and angular brackets $\langle\cdots\rangle$ denote the configuration averaging. Conductance $G_{12}$ can be calculated for the MA rates [cf. Eq. (1)] according to [36,37]

$$
G_{12}=G_{1} \frac{\exp \left(-\frac{\left|\varepsilon_{1}-\varepsilon_{2}\right|}{2 k_{B} T}\right)}{4 \cosh \left(\frac{\varepsilon_{1}-\varepsilon_{F}}{2 k_{B} T}\right) \cosh \left(\frac{\varepsilon_{2}-\varepsilon_{F}}{2 k_{B} T}\right)}, \quad G_{1}=\frac{e^{2} W_{1}}{k_{B} T},
$$

and the Marcus jump rate [Eq. (2)] yields

$$
G_{12}=G_{2} \frac{\exp \left[-\frac{\left(\varepsilon_{1}-\varepsilon_{2}\right)^{2}}{16 E_{a}^{\text {pol }} k_{B} T}\right]}{4 \cosh \left(\frac{\varepsilon_{1}-\varepsilon_{F}}{2 k_{B} T}\right) \cosh \left(\frac{\varepsilon_{2}-\varepsilon_{F}}{2 k_{B} T}\right)}, \quad G_{2}=\frac{e^{2} W_{2}}{k_{B} T} .
$$

Site energies $\varepsilon_{1}$ and $\varepsilon_{2}$ enter symmetrically into Eq. (7) and (8), and that is why $\sigma_{12}=\sigma_{21}$.

\section{A. Miller-Abrahams nonpolaronic hopping}

Let us first consider the nonpolaronic charge-carrier hopping employing the MA jump rates [Eq. (1)]. As it was shown recently [35], the effective conductivity $\sigma_{e}$ within the MA rate model can be calculated using the product of two independent DOS functions, $g\left(\varepsilon_{1}\right) g\left(\varepsilon_{2}\right)$, for configurational averaging in Eq. (7). The effective MA conductivity is then obtained by substituting Eq. (8) in Eq. (7), and the subsequent configuration averaging yields

$$
\int_{-\infty}^{\infty} \int_{-\infty}^{\infty} d t_{1} d t_{2} \exp \left[-\frac{1}{2}\left(t_{1}^{2}+t_{2}^{2}\right)\right] \frac{\frac{x}{4} \frac{\exp \left(-\frac{x}{2}\left|t_{1}-t_{2}\right|\right)}{\varphi\left(t_{1}, t_{2}, x_{F}\right)}-X_{e}}{\frac{x}{4} \frac{\exp \left(-\frac{x}{2}\left|t_{1}-t_{2}\right|\right)}{\varphi\left(t_{1}, t_{2}, x_{F}\right)}+2 X_{e}}=0 .
$$

Here $\quad X_{e}=\sigma_{e} / \sigma_{1}, \quad \sigma_{1}=e^{2} W_{1} / a \sigma, \quad \phi\left(t_{1}, t_{2}, x_{F}\right)=$ $\cosh \left[(x / 2)\left(t_{1}-x_{F}\right)\right] \cosh \left[(x / 2)\left(t_{2}-x_{F}\right)\right], \quad x=\sigma / k_{B} T$, and $x_{F}=\varepsilon_{F} / \sigma$.

Figure 1(a) (bold curves) shows the temperature-dependent effective nonpolaronic conductivity $\sigma_{e} / \sigma_{1}$ calculated by Eq. (10) for different carrier concentrations using MA rates. Thin lines in Fig. 1(a) are linear asymptotes to the above calculated Arrhenius plots (bold lines) made in the temperature range where the $\ln \left(\sigma_{e} / \sigma_{1}\right) \propto 1 / T$ law is obeyed, and these asymptotes can be parameterized by the following approximate analytical relation

$$
\begin{aligned}
\frac{\sigma_{e}^{a s}}{\sigma_{1}}= & \exp \left\{1.4-\left(\frac{\sigma}{k_{B} T}+1\right)[0.385-1.121\right. \\
& \left.\left.\times \log \left(\frac{n}{N}\right)-0.0854 \times \log ^{2}\left(\frac{n}{N}\right)\right]\right\} .
\end{aligned}
$$

As one can note, these asymptotes obtained at different carrier concentrations feature a single crossing point at a "negative" temperature, which is reminiscent of the so-called anti-MN rule (or inverted MN rule) behavior, with negative $E_{\mathrm{MN}}$ energy observed before for the temperature-dependent conductivity in heavily doped microcrystalline Si $[26,38]$ and some a-Si devices [39]. According to the present calculations, the isokinetic temperature $T_{\mathrm{MN}}$ in Fig. 1(a) depends on the
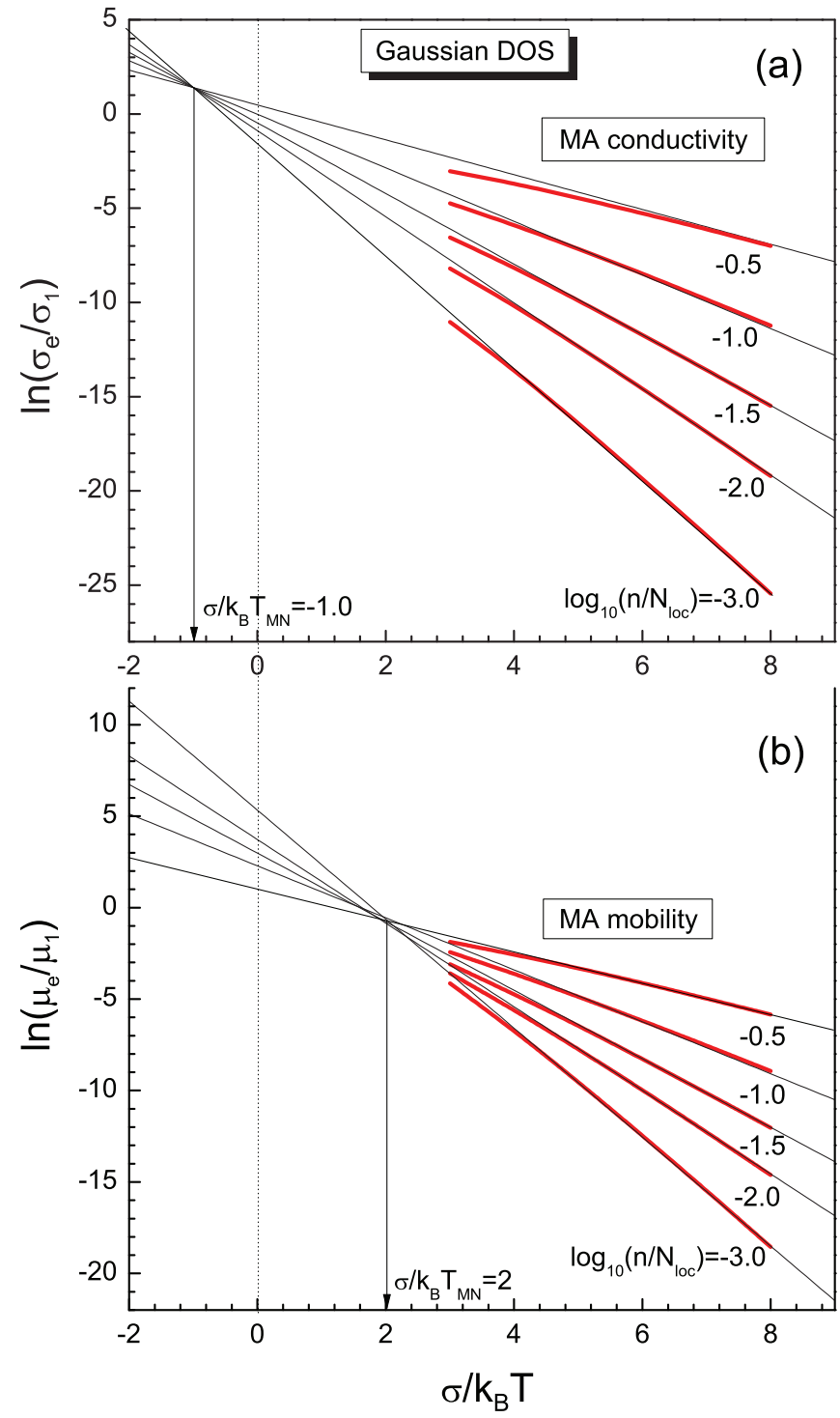

FIG. 1. (Color online) Temperature dependences of (a) the effective nonpolaronic conductivity $\sigma_{e} / \sigma_{1}$ and (b) mobility $\mu_{e} / \mu_{1}$ calculated for a Gaussian DOS and MA jump rates at different charge-carrier concentrations $(n / N)$ (red bold curves). Thin straight lines represent the approximated dependences calculated by Eq. (11). Vertical dashed line denotes $\sigma / k_{B} T=0$ and is a guide to the eye.

width of the DOS as $\sigma / k_{B} T_{\mathrm{MN}}=-1.0$, and it thus allows estimating the $\sigma$ from experimentally observed $T_{\mathrm{MN}}$.

The effective nonpolaronic mobilities were calculated from the above conductivity data [cf. Fig. 1(a)] using Eq. (6) as $\mu_{e} / \mu_{1}=(N / n) \sigma_{e} / \sigma_{1}$, where $\mu_{1}=e a^{2} W_{1} / \sigma$, and the obtained temperature dependences are presented in Fig. 2(b) (bold curves). The asymptotes to the calculated $\mu_{e}(T)$ dependences also feature a MN compensation behavior upon varying the carrier concentration. However, in contrast to the conductivity, the crossing point $T_{\mathrm{MN}}$ (isothermal temperature) is positive for the mobilities, which is fully in line with previous papers when the charge-carrier mobility was calculated directly [15]. The latter fact verifies the validity of the present approach, and therefore it is natural that these results are similar to those we obtained before for MA mobilities [15]. 

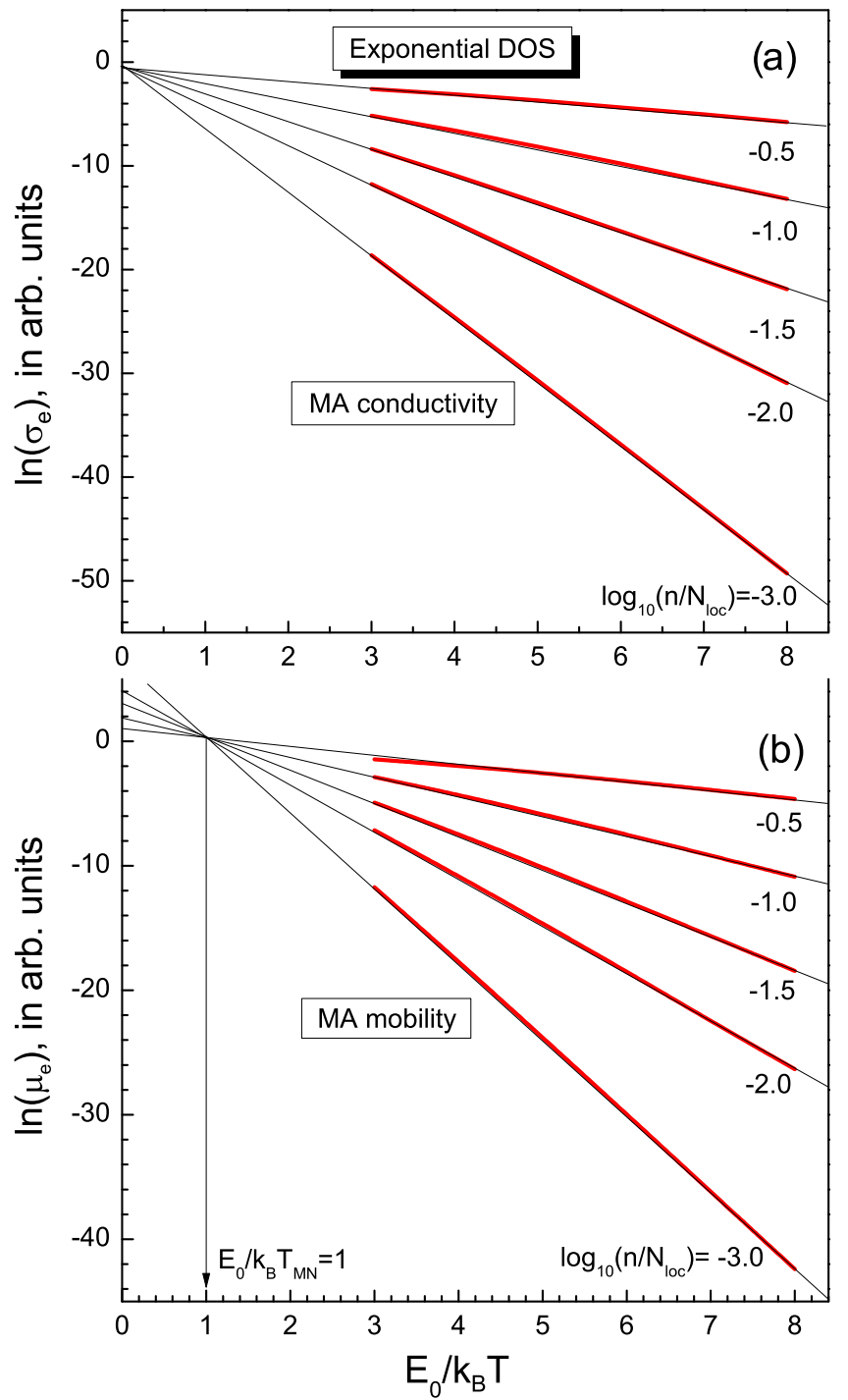

FIG. 2. (Color online) Temperature dependences of (a) the effective nonpolaronic conductivity $\sigma_{e}$ and (b) mobility $\mu_{e}$ calculated for an exponential DOS and MA rates at different charge-carrier concentrations $(n / N)$ (red bold curves). Thin straight lines are linear approximations of the calculated data in an Arrhenius representation.

It would be of interest to figure out how the DOS function profile can affect the MN behavior. Below, we compare the above calculations obtained for the Gaussian DOS with those for an exponential DOS distribution, which is also sometimes applied to organic semiconductors [40]. The same analytical formalism was therefore applied to consider the nonpolaronic hopping transport in an exponential DOS distribution
$g(\varepsilon)=N / E_{0} \times \exp \left(\varepsilon / E_{0}\right)(-\infty<\varepsilon \leqslant 0)$, where $E_{0}$ is the width of the exponential distribution, and the calculation results are presented in Fig. 2. The calculated Arrhenius plots demonstrate a $\mathrm{MN}$ behavior for the temperaturedependent mobilities [Fig. 2(b)], featuring a crossing point at $E_{0} / k_{B} T_{\mathrm{MN}}=1$ for the corresponding linear asymptotes, while no $\mathrm{MN}$ behavior is found for the temperature dependences of the conductivity [Fig. 2(a)], which intersect at infinite temperature. It should be noted that these isothermal temperatures are found to be exactly the same as reported in a relevant paper by Vissenberg and Matters [40], who applied a percolation theory to the charge-carrier hopping in an exponential DOS. The fact that the result of Ref. [40] has been recovered using the present EMA method testifies to the applicability of our theoretical approach.

Comparison of the calculation results in Figs. 1 and 2 proves that the shape of the DOS distribution has a significant effect on the MN behavior. The Gaussian DOS is certainly a good and most commonly accepted approximation for organic materials. However, recently it was suggested that the DOS profile in real organic disordered organic semiconductors could not be of perfectly Gaussian shape, but rather it has the form

$$
g(\varepsilon) \propto \exp \left[-\frac{1}{p}\left(\frac{\varepsilon}{\sigma}\right)^{p}\right],
$$

where $p>1.8$ was needed to explain experimental results

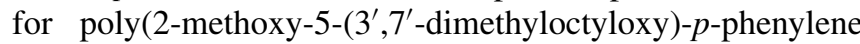
vinylene $\left(\mathrm{OC}_{1} \mathrm{C}_{10}-\mathrm{PPV}\right)$ and poly-3-hexylthiophene (P3HT) [41]. Generally, $p$ values might range within $1 \leqslant p \leqslant 2$ [41]. In such situations the $\mathrm{MN}$ isokinetic temperatures observed experimentally might somewhat differ, and therefore the MN effect might behave in an intermediate manner to what is predicted for pure Gaussian and pure exponential DOS shapes.

\section{B. Marcus polaronic hopping}

Next, we consider polaronic hopping transport within a Gaussian DOS using the Marcus rate [Eq. (2)]. Recently it was demonstrated [35] that appropriate configurational averaging procedure has a profound effect on calculation results using Eq. (6) for the Marcus hopping transport. It was shown that results consistent with Monte-Carlo simulations for the Marcus hopping, when both disorder and polaronic effects are simultaneously present at arbitrary $E_{a}^{\mathrm{pol}} / \sigma$ ratio, can be obtained when configurational averaging uses a product of functions $g\left(\varepsilon_{1}\right) f\left(\varepsilon_{1}, \varepsilon_{F}\right) g\left(\varepsilon_{2}\right)\left[1-f\left(\varepsilon_{2}, \varepsilon_{F}\right)\right]$ [35]. Applying this averaging procedure in Eq. (7), and using Eqs. (6) and (8), one obtains the following transcendental equation

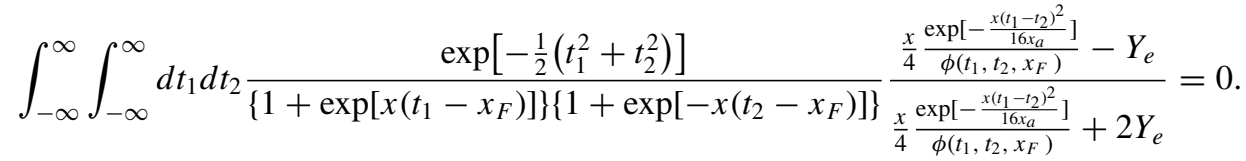

Here $\quad Y_{e}=\sigma_{e} / \sigma_{2}, \quad \sigma_{2}=e^{2} W_{2} / a \sigma, \varphi\left(t_{1}, t_{2}, x_{F}\right)=$ $\cosh \left[(x / 2)\left(t_{1}-x_{F}\right)\right] \cosh \left[(x / 2)\left(t_{2}-x_{F}\right)\right], \quad$ and $\quad x_{a}=$ $E_{a}^{\mathrm{pol}} / \sigma$.
Temperature dependences of the effective chargecarrier mobility $\mu_{e} / \mu_{2}=(N / n) Y_{e} \exp \left(-x x_{a}\right)$, where $\mu_{2}=$ $e a^{2} W_{2} / \sigma$, calculated for a Gaussian DOS and Marcus rates 

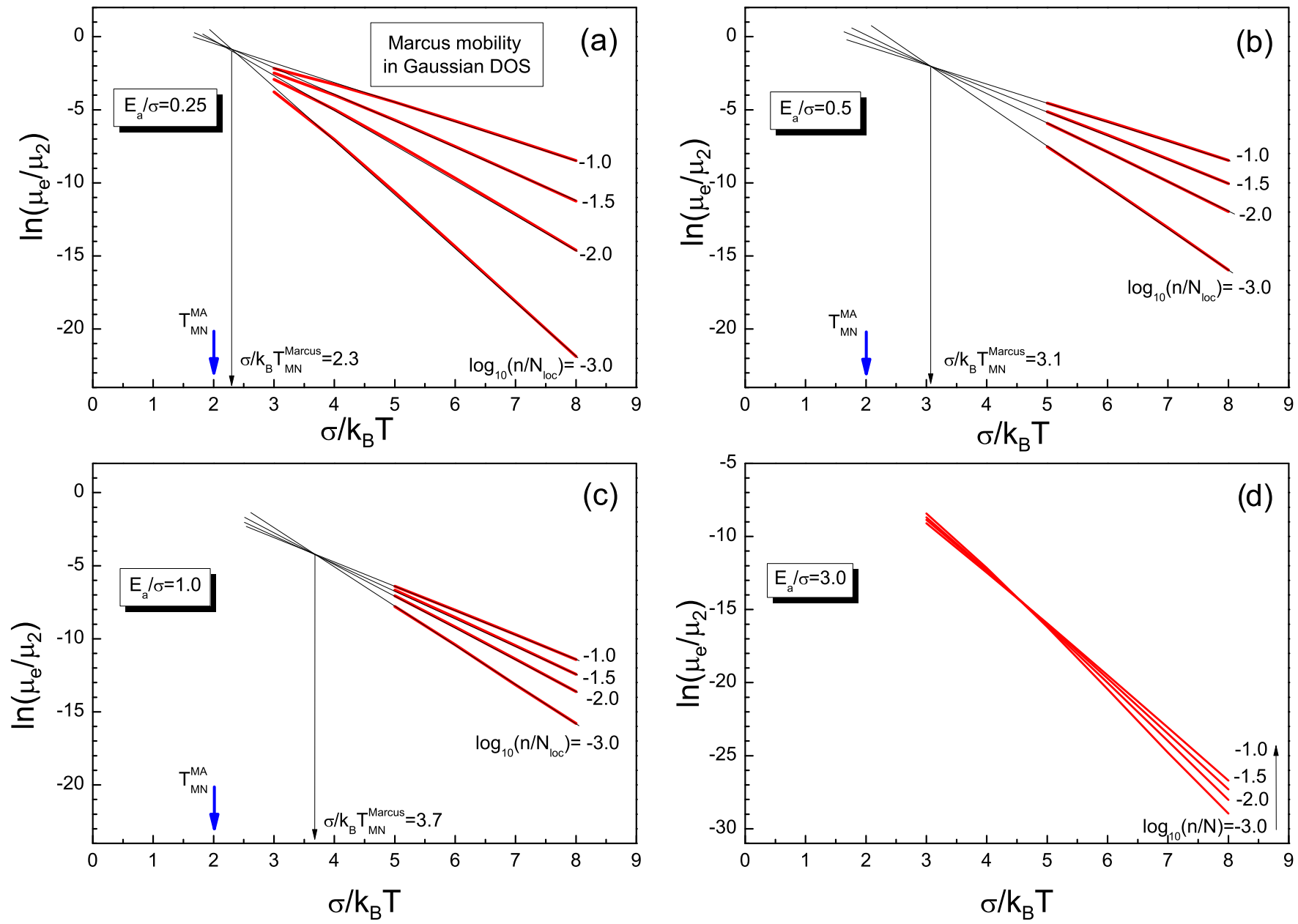

FIG. 3. (Color online) Temperature dependences of the effective polaronic charge-carrier mobility calculated for Marcus rates as $\mu_{e} / \mu_{2}=$ $(N / n)\left(\sigma_{e} / \sigma_{2}\right) \exp \left(-x x_{a}\right)$ for different ratios between polaron activation energy and the width of the DOS with $E_{a}^{\mathrm{pol}} / \sigma$ equal to (a) 0.25 , (b) 0.5 , (c) 1 , and (d) 3 . Note that the calculated curves practically merge at large $E_{a}^{\mathrm{pol}} / \sigma \geqslant 3$ ratios (d), so no MN behavior could be clearly distinguished. Short bold arrows indicate the isokinetic temperature $T_{\mathrm{MN}}$ obtained for the nonpolaronic charge mobility calculated for MA jump rate [cf. Fig. 2(b)] and are shown for reference.

using Eqs. (6) and (13) at different carrier concentrations are shown in Fig. 3 by red bold curves for different ratios $E_{a}^{\mathrm{pol}} / \sigma=0.25,0.5,1$, and 3 (indicated in figure). Asymptotes to the calculated curves are given by black thin lines, and at relatively small $E_{a}^{\mathrm{pol}} / \sigma$ ratios, they are found to feature a MN compensation behavior with an intersection point at a finite temperature, which turns out to depend on the $E_{a}^{\mathrm{pol}} / \sigma$ ratio [Figs. 3(a)-3(c)].

Note that when polaron effects decrease, i.e., the $E_{a}^{\mathrm{pol}} / \sigma$ ratio gets smaller, the isokinetic $\mathrm{MN}$ temperature for polaronic mobility $T_{\mathrm{MN}}^{\text {Marcus }}$ approaches that obtained for nonpolaronic MA mobility, $T_{\mathrm{MN}}^{\mathrm{MA}}$, indicated in Fig. 3 by the short arrow for comparison. This additionally justifies that the MA jump rate model can indeed provide an adequate description for the charge hopping transport in conventional organic semiconductors with small polaron effects.

\section{COMPARISON WITH EXPERIMENTAL RESULTS}

To verify the present model, we performed a test experiment where we compared the MN behavior for conductivity and charge mobility $\mu(T)$ measured in an OFET device based on pentacene. The latter is often considered as a benchmark organic semiconductor with relatively small polaron effects [8], and charge-carrier mobility properties were described successfully with the EGD model [18] using MA rates. We fabricated OFET devices with pentacene functional films in a vacuum deposited onto a $\mathrm{SiO}_{2}(120 \mathrm{~nm})$ dielectric pretreated with a poly $(\alpha$-methylstyrene) (P $\alpha \mathrm{MS})$ layer optimized to maximize pentacene crystallinity (experimental details were already published elsewhere [42]). Top contacts (Au) were then deposited to define transistor structures for temperaturedependent measurements. No permanent device degradation was observed when comparing room temperature measurements before and after the cooling cycle. At moderately high temperatures (300-150 K), the OFET devices were found to exhibit thermally activated conductivity and mobilities.

Arrhenius plots of temperature-dependent source-drain $\left(I_{\mathrm{SD}}\right)$ current and the charge-carrier mobility $(\mu)$ measured at different gate voltages $V_{\mathrm{GS}}$ in the pentacene-based OFET device are presented in Figs. 4(a) and 4(b) (symbols), respectively. Measurements were done in the linear regime of 

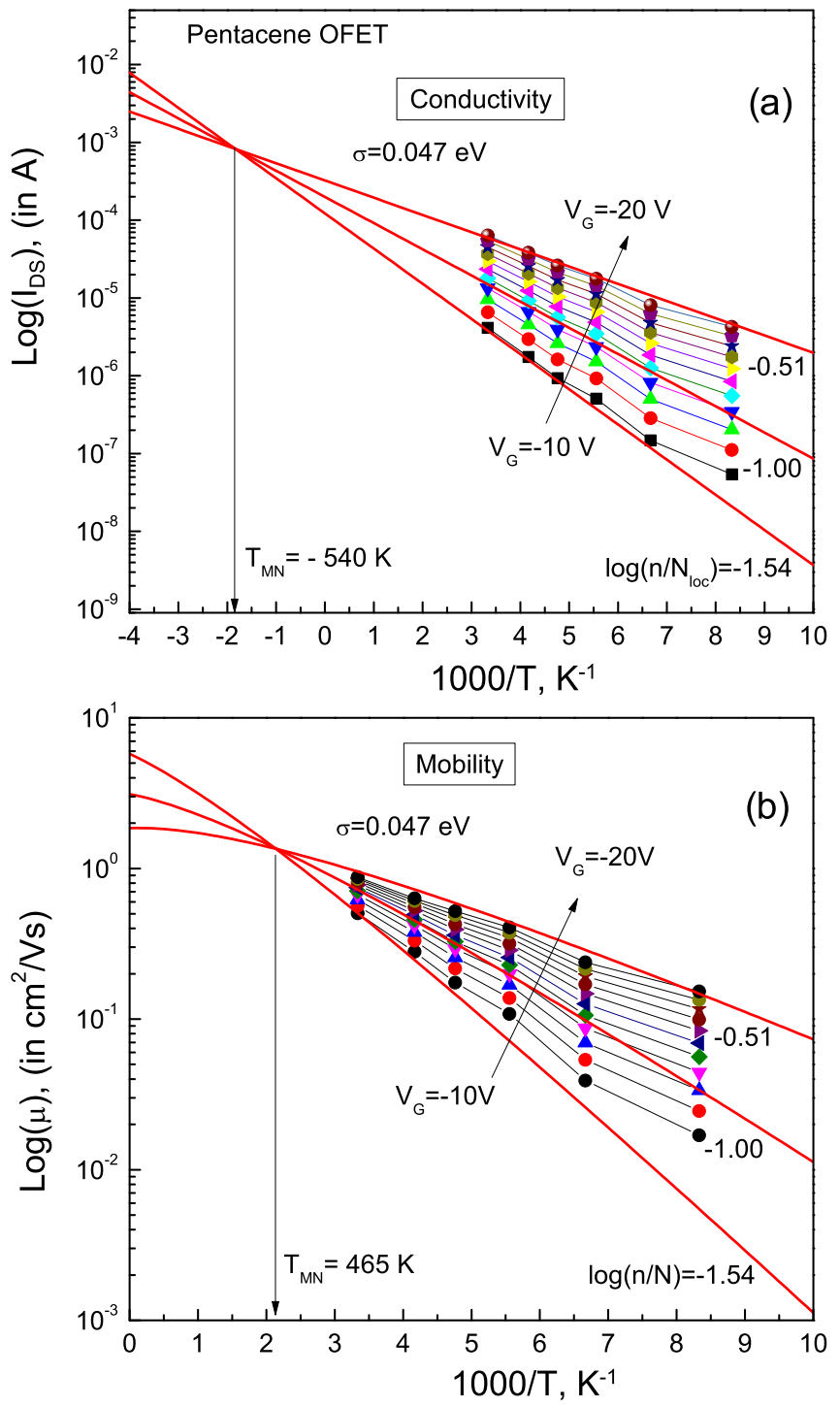

FIG. 4. (Color online) Temperature dependence of (a) the $I_{\mathrm{SD}}(T)$ and (b) OFET mobility $\mu(T)$ in a pentacene film measured at different $V_{\mathrm{GS}}$ voltages (symbols) and results of their theoretical description with the present model (red solid curves). The isothermal temperature is indicated by a vertical arrow (note that it is negative for conductivity and positive for mobility).

the $I_{\mathrm{SD}}-V_{\mathrm{GS}}$ transfer characteristics. We assume here that the current density is proportional to the conductivity as the source-drain voltage was the same in these measurements. Fitting of the experimental $I_{\mathrm{SD}}$ data in Fig. 4(a) was done by Eq. (11) assuming nonpolaronic transport (MA rates), which is well justified for this material, and shown by solid curves. It is evident that the extrapolations of these graphs intersect at a negative isothermal temperature featuring anti-MN behavior, which perfectly agrees with prediction of the present theory for a Gaussian DOS [cf. Fig. 1(a)]. The theoretical $\mu(T)$ dependences were calculated by differentiating the fitting $I_{\mathrm{SD}}(T)$ curves from the previous ones [Fig. 4(a)] as $\mu \propto \partial I_{d s} / \partial V_{G}$ and are presented in Fig. 1(b) (solid curves). This corresponds to the theoretical relation $\mu_{e}=(1 / e) \partial \sigma_{e} / \partial n$. The $\mu(T)$ curves calculated in such a way describe the experimental results quantitatively reasonably well [Fig. 3(b)], and, in contrast to the conductivity data in Fig. 3(b), the $\mu(T)$ dependences feature a positive isothermal temperature in accordance with the present theory. Thus, both sets of experimental data in Figs. 4(a) and 4(b) can be fitted by the present model using the same material parameters.

We should note that a slight discrepancy in matching the experimental $\mu(T)$ data by dependences calculated using Eq. (6) [Fig. 4(b)] was found to be due to the use of an ideal Gaussian DOS distribution given by Eq. (5). Employment of a recently proposed and probably more realistic DOS function given by Eq. (12) [41] was found to provide a much better fit; however, this makes the whole fitting procedure very complex from a practical point of view. We therefore limit our consideration here to just pure Gaussian DOS [Eq. (5)], and the employment of the DOS in the form of Eq. (12) needs to be investigated carefully in this context.

\section{DISCUSSION}

A key result of the present paper is that $\mathrm{MN}$ behavior regarding the temperature dependences of the charge-carrier mobility and conductivity upon varying the carrier concentration measured in the same OFET material yields distinctly different isokinetic $T_{\mathrm{MN}}$ temperatures for these two related kinetic characteristics (Fig. 3). This can be well rationalized within disorder formalism but is in sharp contrast to MEE model, and therefore the study of the MN compensation behavior for $\mu(T)$ and $I_{\mathrm{DS}}(T)$ dependences at different carrier concentrations can serve as a critical experimental test with which to distinguish between the above models. Indeed, since both $\mu(T)$ and $I_{\mathrm{DS}}(T)$ are governed by the same thermally activated hopping process, the MEE predicts a single characteristic $T_{\mathrm{MN}}$ temperature in the same material because it defines $T_{\mathrm{MN}}$ as the energy of excitations (phonons) divided by a coupling constant $[29,31]$. On the other hand, emergence of different $T_{\mathrm{MN}}$ values for mobility and conductivity is quite expected for the hopping transport in a disordered material with distribution of localized states and is a direct consequence of the presence of energy disorder.

The puzzle can be solved by realizing that the MN compensation behavior observed for the charge transport in OFETs is an apparent extrapolated effect and has nothing to do with occurrence of the genuine $\mathrm{MN}$ rule amenable to interpretation with the MEE model. As was already pointed out in Ref. [15], there is no compensation behavior when the activation energy of the mobility $E_{a}$ is varied by the change of the energetic disorder (the width of the DOS), while it does occur upon varying the carrier concentration (gate voltage), which was verified theoretically and experimentally [20]. This is in clear disagreement with the conventional MN rule, which predicts a correlation between prefactor rate and activation energy regardless of how the change in $E_{a}$ is accomplished, i.e., by either changing the width of the DOS itself or changing the degree of state filling. These arguments imply that for the charge mobility in organic disordered films, there is in fact no genuine correlation between the prefactor and the activated jumps factor, i.e., there can be no change in the attempt of the jump frequency [15]. This seems to be quite natural because otherwise the conventional MN rule would lead to a 
kind of logical contradiction mentioned in Ref. [31] that "the more disordered samples are more conductive than the less disordered samples" due to the fact that the mobility prefactor $\mu_{0}$ would be larger in a more disordered system. Regarding the mobility prefactor, we should recall that, in reality, $\mu(T)$ dependences in organic materials with a Gaussian DOS distribution do not intersect each other. The physical reason is that at high enough temperatures, when the equilibrium energy level shifts beyond the Fermi level, it changes from $\log (\mu) \propto T^{-1}$ to the conventional $\log (\mu) \propto T^{-2}$ dependence (see Ref. [15] for more detailed discussion). Remarkably, the same result was also obtained by computer simulation studies of the charge transport using the numerical master equation method by Coehoorn et al. [12] within the same disorder formalism.

Another result of this paper is it demonstrates that the MA jump rate model yields qualitatively similar compensation behavior as polaronic jump rates based on the Marcus theory, provided that polaron binding energy is not too large, which justifies the MA model applicability and suggests that the multiphonon (polaron) nature of thermally activated jumps is not a prime cause of the MN compensation behavior observed in OFETs. Figure 3 provides clear evidence that the EGD model qualitatively reproduces the MN compensation behavior similarly well for both MA and Marcus mobilities at $E_{a}^{\mathrm{pol}} / \sigma<3$, i.e., when the transport is still dominated by the energy disorder, implying that the polaronic transport in such a disordered system is not much different from the nonpolaronic one. The latter is quite plausible for conventional organic semiconductors like pentacene. The polaron reorganization energy in pentacene is of order of $100 \mathrm{meV}$ [8], implying a polaron activation energy of about $25 \mathrm{meV}$, which is indeed smaller than the value, $\sigma=75-100 \mathrm{meV}$, typically reported for pentacene films $[15,43]$. The isokinetic $T_{\mathrm{MN}}^{\text {Marcus }}$ temperature calculated for the polaronic mobility within Marcus rates clearly tends to approach the $T_{\mathrm{MN}}^{\mathrm{MA}}$ value obtained for the nonpolaronic MA mobility upon decreasing the polaron activation energy (Fig. 3). Thus, reproduction of the compensation behavior is in fact not rate-model-specific, unless the polaron binding energy is relatively very large. A similar conclusion was recently derived [35] from the combined Monte-Carlo simulations and EMA calculations in a study of temperature-dependent hopping diffusivity of triplet excitations in a disordered system with superimposed polaron effects. Indeed, satisfying the detailed balance condition is the major requirement for a rate model to provide adequate description of many transport characteristics in disordered materials, while the choice between Marcus and MA rate models for materials with small or moderate polaron formation energies is not crucial. This proves that the establishment of the compensation behavior in OFETs is a characteristic signature of hopping transport in a random system with variable carrier concentration regardless of the polaronic or nonpolaronic nature of the charge carriers, and, in contrast to the MEE model, multiphonon polaron transfer is not necessary needed to provide the $\mathrm{MN}$ behavior.

The MA rate model has most commonly been used for the analysis of thermally activated charge-carrier hopping transport, and its applicability was proven by extensive computer simulation studies $[3,11,12]$ and analytical treatments $[13,14]$.
Conventionally the MA model is considered as a one-phonon approximation involving only low-frequency acoustic modes; however, this limitation was recently overcome by extending the model to account for all phonon modes, including also high-frequency optical phonons $[44,45]$ in organic semiconductors. Though the polaron Marcus rate model seems to be better justified for organic materials, the results obtained using Marcus and MA rates are in fact not much different in realistic situations [3,4,46-48]. The MA rate is certainly just an approximation when applied at higher temperatures, but its widespread popularity is caused by its simpler mathematical relation and the fact that the numerous parameters of the full polaron model are combined into a single adjustable mobility prefactor parameter. This approximation is based upon tunneling between sites, which is not accounted for in the usual Marcus equation. Consequently, it is unable to explain the decrease of the transition rate when entering the "Marcus inversion region" at high enough electric fields. However, as it was already argued before [47], at realistic material parameters, the inversion does not occur in most organic disordered semiconductors at electric fields below $2 \times 10^{6} \mathrm{~V} / \mathrm{cm}$. Since electronic devices based on disordered organic semiconductors normally operate at $F<2 \times 10^{6} \mathrm{~V} / \mathrm{cm}$, this explains why the MA simplification is equally well applied as the Marcus model to the analysis of charge transport in them.

Let us consider other applicability issues of the MEE model to the MN compensation behavior observed for the chargecarrier transport in OFET devices. The MEE approach [28-31] might look very appealing due to its ambition of universality in explaining the $\mathrm{MN}$ effect in various situations by using rather obvious thermodynamic arguments. However, this does not guarantee that the model can really provide an adequate physical description in all situations, especially if the compensation behavior is an apparent rather than the genuine one. This does not mean that the MEE description is impossible, but it can be of little importance in some situations when other effects prevail, like in disorder-dominated hopping transport system. Several fundamental problems with applying the MEE model to the transport in organic disordered solids have to be admitted:

(i) The principal limitation of the MEE model is that it reduces the complex hopping transport problem in a disordered solid to consideration of just a two-site system - it considers a transition within an individual pair of sites with a fixed difference in site energies [28-31]. In other words, this model attempts to adequately explain charge transport properties in a random system based solely on a transition rate consideration. This is inherently inadmissible for the hopping transport in energetically disordered media. One cannot rule out the relevance of the MEE model, for instance, for a crystalline system where all pairs of sites could be equivalent, e.g., when there is a discrete trap with the same activation energy $E_{a}$. However, in a disordered material, by definition, there is a great variety of such pairs for which energies are described by a DOS distribution, i.e., all pairs of sites have different $E_{a}$. In reality, a charge carrier moving through a random system experiences a large number of hops between sites with very different energies before it reaches a collecting electrode. A prominent example could be the well-known fact that the charge mobility in a random organic system follows the 
$\mu(T) \propto \exp \left[-\left(2 \sigma / 3 k_{B} T\right)^{2}\right]$ dependence [3-7,46-48] in the low-carrier-concentration limit, which cannot be explained just by a hopping rate consideration, but is rather a consequence of carrier motion through a Gaussian-shaped DOS distribution. The same problem is inherent to the polaron model suggested recently by Emin [30], which can be considered as a version of an MEE model [29]: It also never goes beyond the two-site system approximation, which is clearly insufficient and unacceptable to properly treat a disordered system.

(ii) Another piece of evidence for inapplicability of the MEE model, as well as the related polaron model of Emin [30], to the charge transport in organic semiconductors results from the fact that the compensation behavior arises only when the carrier concentration is sufficiently large, as, e.g., in OFET devices $[12,15,16]$. First, these models clearly fail to explain the dependence of the charge-carrier mobility on carrier concentration (on $V_{G}$ voltage) in OFETs because no DOS tail state filling is expected within the MEE approach. Therefore, there is no charge-carrier concentration factor in these models. For this reason, the MN compensation behavior in OFETs regarding changing the gate voltage cannot be reproduced by these models because they do not predict the dependence of the activation energy on $V_{G}$. Secondly, the Emin theory predicts that the characteristic MN temperature $T_{\mathrm{MN}} \equiv t / k_{B}$ is determined by the electron-transfer energy $t$. Therefore, to rationalize the experimentally observed changes in $T_{\mathrm{MN}}$, which change almost twice with deliberate changing of the energy disorder observed in $\mathrm{C}_{60}$-films grown at different substrate temperatures [20], one has to assume a strong increase of the average intermolecular distances in such films because the Emin model relates the change in parameter $t$ to the change in intermolecular distance. This sounds highly unrealistic and has not been confirmed experimentally. Third, the Fröhlich long-range polaron approach implemented in the Emin model requires a strongly polar medium that is difficult to justify for OFET structures with nonpolar organic semiconductors such as pentacene or $\mathrm{C}_{60}$ and organic dielectric layers. Finally, the Emin model fails to explain why the charge mobility in OFETs with organic single crystal channels is temperature independent or even decreases with temperature in some crystals, while the temperature-dependent charge-carrier transport is typically observed in vapor-deposited thin films of the same material, indicating a key role of disorder effects. Polaron-based models such as the Emin model should result in identical charge transport properties in both cases irrespective of the disorder effects because they attribute the activation energy to polaron formation, which is in clear contradiction to experimental results.

\section{CONCLUSIONS}

Although the MEE model might be applicable to describe the genuine $\mathrm{MN}$ compensation rule in relevant situations where it really happens, including materials with dominant polaron transport, it appears not to be relevant to the charge transport in conventional organic disordered semiconductors with distribution of disordered localized states, and it is contradictory and fails to describe properly experimental observations. Therefore, the recent claim that the MEE concept could provide a possible mechanism for the $\mathrm{MN}$ behavior observed in OFETs is erroneous and misleading. We performed a test experiment based on the combined study of compensation behavior for $\mu(T)$ and $I_{\mathrm{DS}}(T)$ dependences to check applicability of the MEE and EGD models. We found that a distinctly different isothermal temperature $T_{\mathrm{MN}}$ is inherent for the compensation behavior of $\mu(T)$ and $I_{\mathrm{DS}}(T)$ (normal and inverted MN effects, respectively), which is well described by the disorder formalism assuming a Gaussian DOS. In contrast, the MEE model predicts a single $T_{\mathrm{MN}}$ for the same material. The MN compensation behavior observed in OFETs is an apparent effect-it resembles the conventional $\mathrm{MN}$ rule only under certain experimental conditions and arises as a consequence of the functional dependence of state filling of the DOS. This has been validated by both analytic calculations and computer simulations, and it is fully supported by experimental observations. The temperature-dependent mobility in thin-film OFET devices is largely dominated by disorder effects, and small polaron formation plays a minor role. Therefore, the framework of the EGD model provides an adequate description of the $\mathrm{MN}$ compensation behavior in such devices. We demonstrated that the polaron formation can be readily included in this disorder formalism using the Marcus jump rate model, and accounting for moderate polaron formation energies does not significantly alter the qualitative behavior of the charge transport properties, unless the polaron effects become much larger than the disorder ones. The $\mathrm{MN}$ energy $\left(E_{\mathrm{MN}}=k_{B} T_{\mathrm{MN}}\right)$ for the temperature-dependent OFET mobility in a system devoid of polaronic effects is determined by both the energy disorder parameter (the width of the DOS) and shape of the DOS distribution. Therefore, if the realistic DOS profile in an organic semiconductor is not purely Gaussian [41], then $E_{\mathrm{MN}}$ can differ from that predicted for an idealized Gaussian profile and might behave in an intermediate manner to what is predicted for pure Gaussian and pure exponential DOS shapes. Moreover, a moderately large polaron formation energy in disordered systems with significant polaron effects was found to have an additional impact on the $E_{\mathrm{MN}}$ energy.

\section{ACKNOWLEDGMENTS}

The authors gratefully acknowledge valuable discussions with S. V. Novikov (National Research Nuclear University, Moscow). The research was supported by the European Research Council via Grant No. 320680 (EPOS CRYSTALLI) and by the National Academy of Sciences of Ukraine via the program of fundamental research on nanophysics (Project No. 1/14-H-23K). N.S.S. gratefully acknowledges the Austrian Science Foundation (FWF) for funds within the Wittgenstein Prize Z222-N19.
[1] H. Klauk, Organic Electronics: Materials, Manufacturing and Applications (Wiley-VCH, Weinheim, Germany, 2006).
[2] M. Berggren, D. Nilsson, and N. D. Robinson, Nat. Mater. 6, 3 (2007). 
[3] H. Bässler, Phys. Status Solidi B 175, 15 (1993).

[4] P. W. M. Blom and M. C. J. M. Vissenberg, Mater. Sci. Eng. 27, 53 (2000).

[5] H. Bässler and A. Köhler, Top. Curr. Chem. 312, 1 (2012).

[6] P. M. Borsenberger and D. S. Weiss, Organic Photoreceptors for Xerography (Dekker, New York, 1998).

[7] V. I. Arkhipov, I. I. Fishchuk, A. Kadashchuk, and H. Bässler, in Semiconducting Polymers: Chemistry, Physics and Engineering, $2^{\text {nd }}$ ed., edited by G. Hadziioannou and G. Malliaras (Wiley-VCH Verlag, Weinheim, Germany, 2007).

[8] V. Coropceanu, J. Cornil, D. A. da Silva Filho, Y. Olivier, R. Silbey, and J. L. Brédas, Chem. Rev. 107, 926 (2007).

[9] A. Miller and E. Abrahams, Phys. Rev. 120, 745 (1960).

[10] C. Tanase, E. J. Meijer, P. W. M. Blom, and D. M. de Leeuw, Phys. Rev. Lett. 91, 216601 (2003).

[11] W. F. Pasveer, J. Cottaar, C. Tanase, R. Coehoorn, P. A. Bobbert, P. W. M. Blom, D. M. de Leeuw, and M. A. J. Michels, Phys. Rev. Lett. 94, 206601 (2005).

[12] R. Coehoorn, W. F. Pasveer, P. A. Bobbert, and M. A. J. Michels, Phys. Rev. B 72, 155206 (2005).

[13] V. I. Arkhipov, P. Heremans, E. V. Emelianova, G. J. Adriaenssens, and H. Bässler, J. Phys.: Condens. Matter 14, 9899 (2002).

[14] I. I. Fishchuk, V. I. Arkhipov, A. Kadashchuk, P. Heremans, and H. Bässler, Phys. Rev. B 76, 045210 (2007).

[15] I. I. Fishchuk, A. K. Kadashchuk, J. Genoe, M. Ullah, H. Sitter, Th. B. Singh, N. S. Sariciftci, and H. Bässler, Phys. Rev. B 81, 045202 (2010).

[16] E. J. Meijer, E. J. Meijer, M. Matters, P. T. Herwig, D. M. de Leeuw, and T. M. Klapwijk, Appl. Phys. Lett. 76, 3433 (2000).

[17] I. I. Fishchuk, A. K. Kadashchuk, M. Ullah, H. Sitter, A. Pivrikas, J. Genoe, and H. Bässler, Phys. Rev. B 86, 045207 (2012).

[18] X. Li, A. Kadashchuk, I. I. Fishchuk, W. T. T. Smaal, G. Gelinck, D. J. Broer, J. Genoe, P. Heremans, and H. Bässler, Phys. Rev. Lett. 108, 066601 (2012).

[19] W. Meyer and H. Neldel, Z. Tech. Phys. (Leipzig) 18, 588 (1937).

[20] M. Ullah, I. I. Fishchuk, A. K. Kadashchuk, P. Stadler, A. Pivrikas, C. Simbrunner, V. N. Poroshin, N. S. Sariciftci, and H. Sitter, Appl. Phys. Lett. 96, 213306 (2010).

[21] M. Ullah, A. Pivrikas, I. I. Fishchuk, A. Kadashchuk, P. Stadler, C. Simbrunner, N. S. Sariciftci, and H. Sitter, Appl. Phys. Lett. 98, 223301 (2011).

[22] M. Ullah, A. Pivrikas, I. I. Fishchuk, A. Kadashchuk, P. Stadler, C. Simbrunner, N. S. Sariciftci, and H. Sitter, Synth. Met. 161, 1987 (2011).
[23] H. Overhof and P. Thomas, Electronic Transport in Hydrogenated Amorphous Silicon (Springer, Berlin, 1989), p. 122.

[24] S. R. Elliott, Physics of Amorphous Materials, 2nd ed. (Longman Group UK Limited, England, 1990).

[25] T. Drusedau and R. Bindemann, Phys. Stat. Solidi B 136, K61 (1986).

[26] S. K. Ram, S. Kumar, and P. Roca i Cabarrocas, Phys. Rev. B 77, 045212 (2008).

[27] N. F. Mott and E. A. Davis, Electronic Processes in NonCrystalline Materials, 2nd ed. (Oxford University Press, New York, 1979).

[28] A. Yelon and B. Movaghar, Phys. Rev. Lett. 65, 618 (1990).

[29] A. Yelon, B. Movaghar, and R. S. Crandall, Rep. Prog. Phys. 69, 1145 (2006).

[30] D. Emin, Phys. Rev. Lett. 100, 166602 (2008).

[31] A. Yelon, Monatsh. Chem. 144, 91 (2013).

[32] R. A. Marcus, J. Chem. Phys. 24, 966 (1956).

[33] R. A. Marcus, Rev. Mod. Phys. 65, 599 (1993).

[34] V. Kirkpatrick, Rev. Mod. Phys. 45, 574 (1973).

[35] I. I. Fishchuk, A. Kadashchuk, S. T. Hoffmann, S. Athanasopoulos, J. Genoe, H. Bässler, and A. Köhler, Phys. Rev. B 88, 125202 (2013).

[36] V. Ambegaokar, B. I. Halperin, and J. S. Langer, Phys. Rev. B 4, 2612 (1971).

[37] J. Cottaar, L. J. A. Koster, R. Coehoorn, and P. A. Bobbert, Phys. Rev. Lett. 107, 136601 (2011).

[38] S. K. Ram, S. Kumar, and P. Roca i Cabarrocas, J. Non-Cryst. Solids 354, 2263 (2008).

[39] M. Kondo, Y. Chida, and A. Matsuda, J. Non-Cryst. Sol. 198200, 178 (1996).

[40] M. C. J. M. Vissenberg and M. Matters, Phys. Rev. B 57, 12964 (1998).

[41] J. O. Oelerich, D. Huemmer, and S. D. Baranovskii, Phys. Rev. Lett. 108, 226403 (2012).

[42] K. Myny, S. De Vusser, S. Steudel, D. Janssen, R. Müller, S. De Jonge, S. Verlaak, J. Genoe, and P. Heremans, Appl. Phys. Lett. 88, 222103 (2006).

[43] N. G. Martinelli, M. Savini, L. Muccioli, Y. Olivier, F. Castet, C. Zannoni, D. Beljonne, and J. Cornil, Adv. Funct. Mater. 19, 3254 (2009).

[44] N. Vukmirovic and L.-W. Wang, Appl. Phys. Lett. 97, 043305 (2010).

[45] N. Vukmirovic and L.-W. Wang, NANO Lett. 9, 3996 (2009).

[46] S. V. Novikov, D. H. Dunlap, V. M. Kenkre, P. E. Parris, and A. V. Vannikov, Phys. Rev. Lett. 81, 4472 (1998).

[47] P. E. Parris, V. M. Kenkre, and D. H. Dunlap, Phys. Rev. Lett. 87, 126601 (2001).

[48] M. A. Baldo and S. R. Forrest, Phys. Rev. B 64, 085201 (2001). 